\title{
Liquid Crystalline Cyclotetraphosphazene
}

\author{
Derya Davarc1*, Ceylan Mutlu Balc1 \\ Department of Chemistry, Faculty of Science, Gebze Technical University, Gebze, Turkey \\ *ddavarci@gtu.edu.tr
}

Received: 8 October 2017

Accepted: 15 May 2018

DOI: $10.18466 /$ cbayarfbe. 350047

\begin{abstract}
Full substituted dodecyloxy derivative of cyclotetraphosphazene was synthesized in order to investigate its mesomorphic properties. Octachlorocyclotetraphosphazene (tetramer) was reacted with dodecanol in tetrahydrofuran (THF) presence of sodium hydride $(\mathrm{NaH})$ as base at room temperature for 24 hours and then under the reflux for a day. Full substituted derivative (1) was obtained and its structure was determined by mass analysis, and by FT-IR, ${ }^{1} \mathrm{H}$ and ${ }^{31} \mathrm{P}\left\{{ }^{1} \mathrm{H}\right\}$ NMR spectroscopic techniques. Additionally, thermal and mesomorphic behaviours were examined by differential scanning calorimetry (DSC), polarizing optical microscopy (POM) and X-ray diffraction (XRD) techniques. Obtained results showed that compound $\mathbf{1}$ has liquid crystal behaviour.

Keywords: Cyclotetraphosphazene; Liquid crystal; Differential scanning calorimeter; Polarising optical microscopy; X-ray Diffraction.
\end{abstract}

\section{Introduction}

Liquid crystals are significant class of compounds and play an important role in the area of technological applications such as displayers, temperature maps, electrical, optical, and switching materials [1-2]. Cyclophosphazenes are important starting materials because of having multiarmed rigid ring and their active $\mathrm{P}-\mathrm{Cl}$ bounds so, they can easily replace a lot of reagent, for example organic, inorganic and organometallic reactive, by the nucleophilic substitution reaction. Much attention has been focused on several studies of cyclophosphazenes because they acquire different physical properties with different side chains. In addition, in the field of liquid crystals, cyclotriphosphazene (trimer) and cyclotetraphosphazene (tetramer) (Figure 1) have been studied. Some trimeric derivatives showed liquid crystallinity [3-15]. Mesomorphic behaviour of tetramer was studied by Moriya et al, and some phase transition was seen for octacis-[4'-(4-octyloxy) biphenoxy] cyclotetraphosphazene and octacis- $\{[4-[\mathrm{N}-(4$, heptylyloxphenyl)iminomethyl]phenoxy] cyclotetraphos- $^{-}$

phazene [16]. Molecular shape is an important factor in the formation of liquid crystal molecules. Generally, mesogenic units having one terminal chain on the aromatic ring gives rise to calamitic mesomorphism, whereas the presence of a large number of terminal chains on the aromatic ring causes the molecule to adopt a discotic structure $[3,4$, $14,15]$.<smiles>ClP1(Cl)=NP(Cl)(Cl)=NP(Cl)(Cl)=NP(Cl)(Cl)=N1</smiles>

Figure 1. Structure of Cyclotetraphosphazene

Calamitic mesomorphism has been reported previously for cyclophosphazene derivatives and this behaviour was explained in terms of a model consisting of mesogenic units arranged approximately perpendicular to the cyclophosphazene ring [5-13]. In this study, tetramer was selected as starting material and dodecyloxy derivative of cyclotetraphosphazene (1) was synthesized (Scheme1). Than its liquid crystal behaviour examined by POM, DSC and XRD techniques. Results show that compound $\mathbf{1}$ has liquid crystal phase transition and it is a liquid crystal molecule.

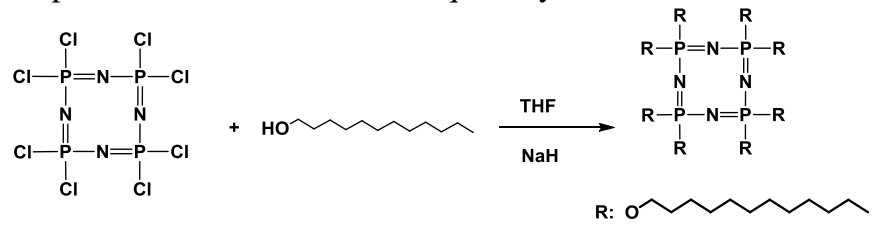

Scheme 1. Reaction of tetramer with dodecanol. 


\section{Materials and Methods}

\subsection{Instruments}

Elemental analyses were obtained using a Thermo Finnigan Flash EA 1112 series, Italy instrument. Mass analyses were recorded on a Bruker MALDI-TOF (Matrix-Assisted Laser Desorption/Ionization-Time-Of-Flight mass, Rheinstetten, Germany) spectrometer for compound 1 (using 2,5-dihydroxybenzoic acid Merck, Darmstadt, Germany as matrix). Fourier transform infrared spectroscopy (FT-IR) spectra were recorded with Perkin Elmer Spectrum 100 spectrometer (Perkin Elmer, Waltham, MA, USA). ${ }^{1} \mathrm{H}$ and ${ }^{31} \mathrm{P}\left\{{ }^{1} \mathrm{H}\right\}$ NMR spectra (Varian Inova, West Sussex, UK) were recorded in $\mathrm{CDCl}_{3}$ solutions on a Varian INOVA $500 \mathrm{MHz}$ spectrometer. Differential Scanning Calorimeter (DSC) measurement of compound 1 was performed on a Mettler Toledo DSC 821 instrument (Mettler Toledo, Columbus, $\mathrm{OH}$, USA) under a protective nitrogen atmosphere from $40{ }^{\circ} \mathrm{C}$ to $100{ }^{\circ} \mathrm{C}$ at a heating/cooling rate of $10^{\circ} \mathrm{Cmin}^{-1}$. Polarising optical microscopy (POM) was performed using a Leica Wild MPS52 polarizing microscope equipped with Linkam TMS 93 heating and Linkam LNP cooling unit (Leica, Heerbrugg, St. Gallen, Switzerland). Powder X-ray diffraction (XRD) patterns were recorded at room temperature up to resolution of $2 \Theta=2.0-25^{\circ}$ on a Rigaku X-ray diffractometer D-Max Rint 2200 Series instrument using $\mathrm{Cu} \mathrm{K} \alpha$ radiation at $40 \mathrm{kV}$ and $40 \mathrm{~mA}$.

\subsection{Chemicals}

Tetramer was obtained from Otsuka Chemical Co., Ltd. and purified by fractional crystallisation from $n$-hexane. $\mathrm{NaH}$ (Merck, 60\% dispersion in mineral oil); prior to use the oil was removed by washing with dry $n$-hexane followed by decantation. Tetrahydrofuran (THF), hexane, dichloromethane and dodecanol were obtained from Merck and used as received. Silica gel (Merck, Kieselgel 60, 70-230 mesh) was used for column chromatography. Reactions were peformed under a dry argon atmosphere.

\subsection{Reaction of tetramer with dodecanol to prepare compound 1}

Dodecanol (4.7 g, $25 \mathrm{mmol})$ was dissolved in $30 \mathrm{~mL}$ of dry THF in a $250 \mathrm{~mL}$ three-necked round-bottom flask. NaH (60\% oil suspension, $1 \mathrm{~g}, 25 \mathrm{mmol}$ ) in $20 \mathrm{~mL}$ of dry THF was quickly added to the stirred solution under an argon atmosphere. Tetramer (1.16 g, $2.5 \mathrm{mmol})$ dissolved in 20 $\mathrm{mL}$ of THF was added dropwise under an argon atmosphere. The reaction was stirred for a further $24 \mathrm{~h}$ at room temperature and then under the reflux for a day followed by TLC on silica gel plates using hexane-dichloromethane $(1: 1)$ as the mobile phase. The reaction mixture was filtered to remove the sodium chloride and any other insoluble material. The solvent was removed under reduced pressure and the crude product was subjected to column chromatography using hexane-dichloromethane (1:1) as eluent. The octadodecyloxy substituted compound (1) was isolated.

\section{Result and Discussion 3.1. Characterization}

The structure of compound $\mathbf{1}$ was characterised by mass and elemental analyses, and FT-IR, ${ }^{1} \mathrm{H}$ and ${ }^{31} \mathrm{P}\left\{{ }^{1} \mathrm{H}\right\}$ NMR spectra. Mass spectrum of compound 1 was showed in Figure $2(1662.88 \mathrm{~m} / \mathrm{z})$. FT-IR spectrum of $\mathbf{1}$ displayed that characteristic $\mathrm{C}-\mathrm{H}$ aliphatic stretching vibrations in the range of $2917-2846 \mathrm{~cm}^{-1}$ and the vibration bands assignable to the stretching of the $\mathrm{P}-\mathrm{N}$ and $\mathrm{P}-\mathrm{O}$ for compound $\mathbf{1}$ were observed at frequency in the range of $1284 \mathrm{~cm}^{-1}$ and $1064 \mathrm{~cm}^{-1}$ (Table 1).

Table 1. Selected FT-IR vibrations of $\mathbf{1}\left(\mathrm{cm}^{-1}\right)$.

\begin{tabular}{|c|c|c|c|c|}
\hline No & $\begin{array}{c}\mathbf{V} \\
(\mathbf{C}-\mathbf{H})\end{array}$ & $\begin{array}{c}\mathbf{v} \\
(\mathbf{C}-\mathbf{C})\end{array}$ & $\begin{array}{c}\mathbf{v} \\
(\mathbf{P}-\mathbf{N})\end{array}$ & $\begin{array}{c}\mathbf{V} \\
(\mathbf{P}-\mathbf{O})\end{array}$ \\
\hline $\mathbf{1}$ & 2917, & 1501 & 1284 & 1064 \\
& 2846 Vs & S & S & S \\
\hline
\end{tabular}

br: broad; s: strong; vs: very strong; m: medium ${ }^{1}$ aliphatic vibrations

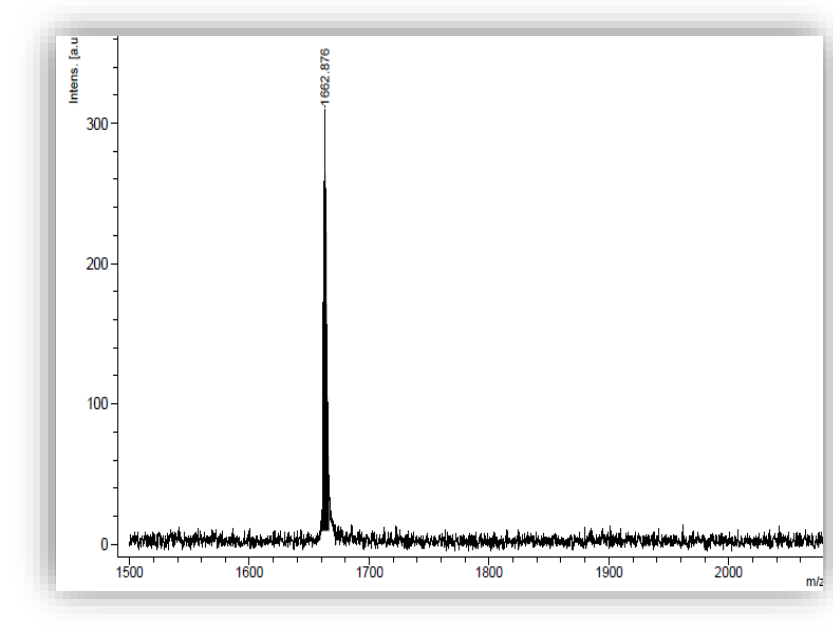

Figure 2. Mass spectrum of compound $\mathbf{1}$.

The proton decoupled ${ }^{31} \mathrm{P}$ NMR spectrum of compound $\mathbf{1}$ showed only a single peak ( $\mathrm{A}_{4}$ spin systems) at $0.75 \mathrm{ppm}$ because of equal environments of the phosphorus nucleus (Fig 3). The ${ }^{1} \mathrm{H}$ NMR spectra of compound 1 showed the chemical shifts for $\mathrm{CH}_{2}$ protons adjacent to $\mathrm{O}\left(-\mathrm{OCH}_{2}-\right)$ were observed at $3.88-3.91 \mathrm{ppm}(\mathrm{m}, 2 \mathrm{H}) ;-\mathrm{CH}_{3}$ protons at 0.87-0.90 ppm (t, 3H); $-\mathrm{OCH}_{2} \mathrm{CH}_{2}-1.60-1.65 \mathrm{ppm}(\mathrm{m}, 2 \mathrm{H})$ and other $\mathrm{CH}_{2}$ 1.26-1.34 ppm $(\mathrm{m}, 18 \mathrm{H}) .{ }^{1} \mathrm{H}$ NMR spectrum of compound 1 was given in Figure 4. 


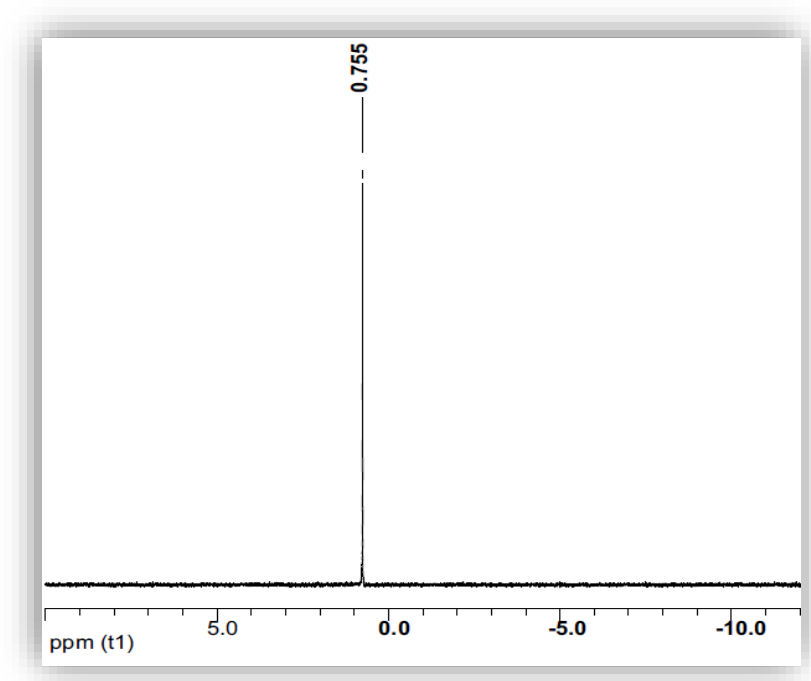

Figure 3. ${ }^{31} \mathrm{P}\{\mathrm{H}\}$ NMR spectrum of compound 1 .

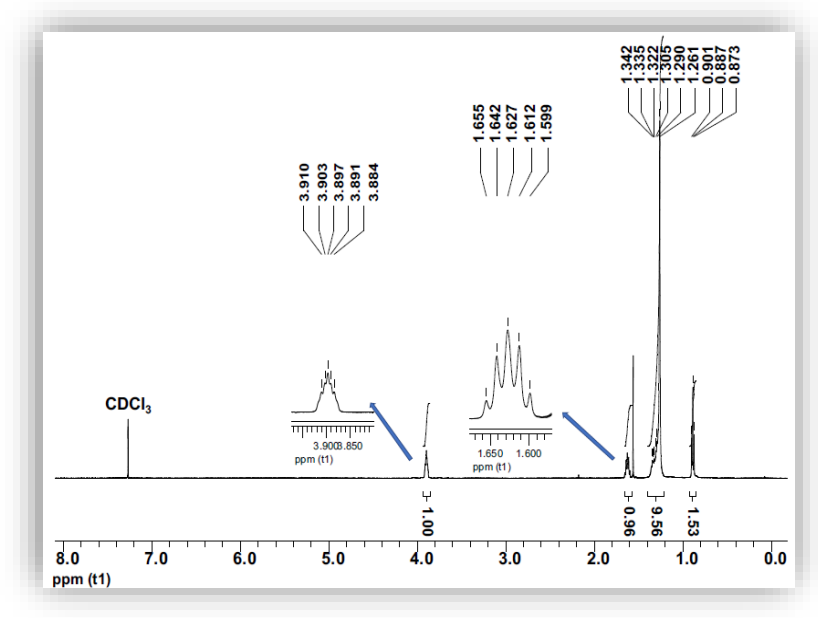

Figure 4. ${ }^{1} \mathrm{H}$ NMR spectrum of compound $\mathbf{1}$.

\subsection{Mesomorphic Properties}

The mesomorphism of compound $\mathbf{1}$ was studied by DSC, $\mathrm{POM}$ and XRD techniques. The compound gave reproducible DSC curves for heating and cooling over a few cycles and typical thermogram for the third heating and cooling processes was given in Figure 5. Compound 1 showed two endothermic peaks in the heating cycle of the DSC thermograms at $-12.10^{\circ} \mathrm{C}$ and at $42.09^{\circ} \mathrm{C}$. In the cooling cycle, 1 has two exothermic peaks which were observed at $34.23{ }^{\circ} \mathrm{C}$ and $-21.23{ }^{\circ} \mathrm{C}$. (Fig.5). (The first endothermic peak at $12.10^{\circ} \mathrm{C}$ belongs to a crystal-liquid crystalline transition; the second endothermic peak at $42.09^{\circ} \mathrm{C}$ belongs to a liquid crystalline-izotropic liquid transition). Enthalpy changes $(\Delta \mathrm{H})$ for all transition $4.47,188.36,168.71,6.77 \mathrm{kJmol}^{-1}$.

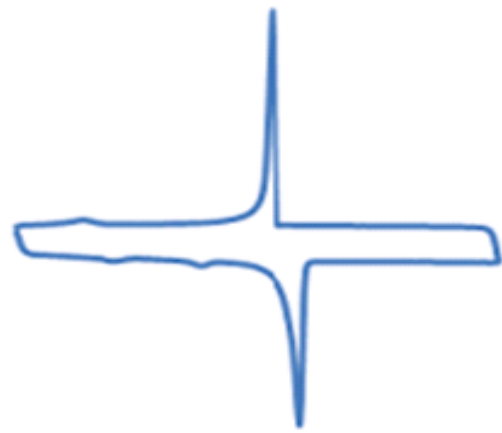

Figure 5. DSC termograms of compound $\mathbf{1}$.

The sample for POM investigation was prepared as a thin film between two glass plates by melting before visual inspection and the POM image for compound $\mathbf{1}$ was shown in Figure 6. POM investigating of compound 1 supported results of DSC measurements. During the heating process, compound $\mathbf{1}$ initially appeared as liquid crystal textures under the microscope and the basic texture remained unchanged until the isotropic phase transition. During the cooling process, the same texture occurred again on each side of the film. Although the texture image of $\mathbf{1}$ was protected until room temperature, below the crystal-liquid crystal transition temperatures, the fluidity of these materials was lost and a cracked zone took place on the film surfaces by mechanical stress. Images of liquid crystal texture for the compound $\mathbf{1}$ was focal conic or broken fan-like which were reminiscent of smectic liquid crystals [12. 17].

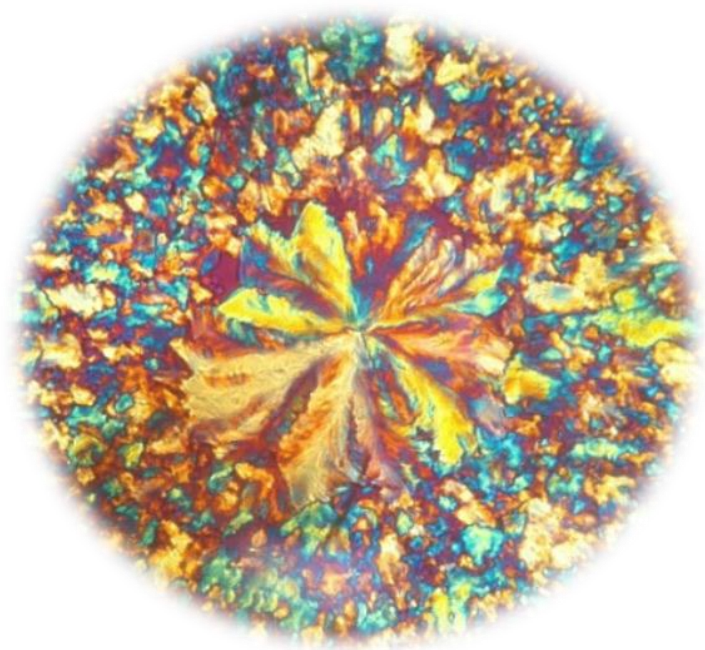

Figure 6. POM image of compound $\mathbf{1}$ (magnification 40x).

The compound was also investigated by powder X-ray diffraction (XRD) at room temperature in order to gain more information on the organization of layers. For this purpose, 
thermal treated film sample was prepared for XRD analysis. The XRD patterns of the compound $\mathbf{1}$ had a strongsharp peak in the small angle region (SAXS) at $2 \theta=2.62^{\circ}$. This peak indicated that the molecules arranged in regularly spaced layers with the layer distance depended on length of terminal chains as $33.70 \AA$ for compound $\mathbf{1}$. In addition, the broad diffraction bands observed at $2 \theta=20-25^{\circ}$ and in the wide angle region (WAXS) implied the disordered packing of melt hydrophobic alkyl chains in the layer [12,18-20]. XRD result supported liquid crystallinity of $\mathbf{1}$ and XRD diffractogram of compound $\mathbf{1}$ was shown in Figure 7.

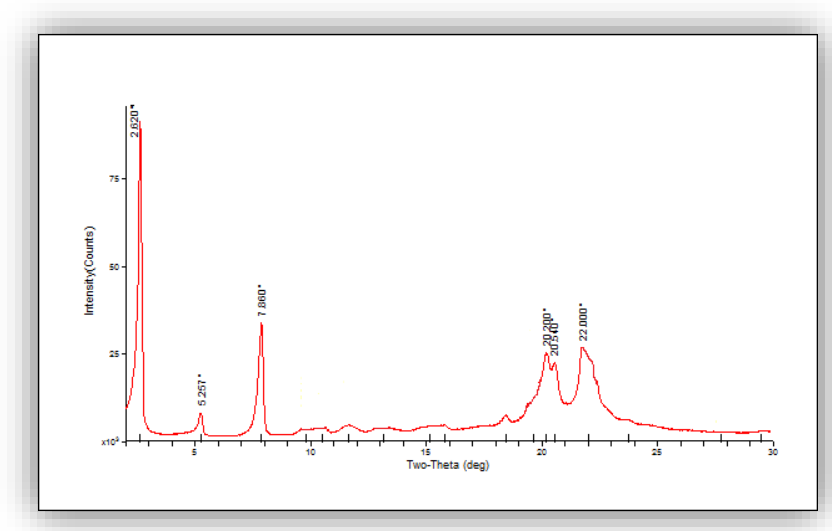

Figure 7. XRD diffractogram of compound 1.

In compound $\mathbf{1}$, side chains connected to the $\mathrm{P}$ atoms of the tetramer ring are arranged four up and four down, perpendicularly to the ring. This shape is suitable for smectic arrangement and its possible arrangement was illustrated in Figure 8.

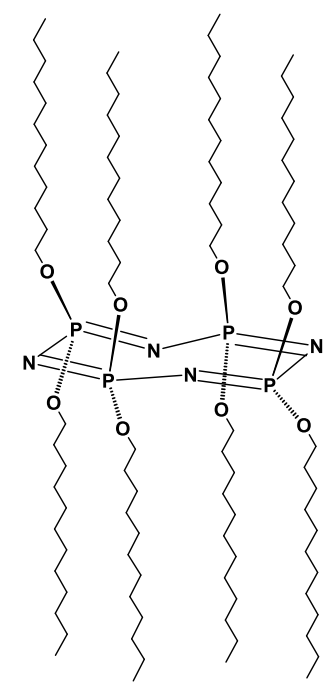

Figure 8. Molecular shape of compound 1.

\section{Conclusion}

Octacis(p-dodecyloxy)cyclotetraphosphazene (1) was synthesized and fully characterized by standard spectroscopic techniques. Liquid crystallinity of compound $\mathbf{1}$ was confirmed by POM, DSC and XRD techniques. Information on the organization of layers of the compound was obtained by $\mathrm{XRD}$ technique. Results showed that compound $\mathbf{1}$ is liquid crystal and has mesomorphic properties. Compound $\mathbf{1}$ might be a suitable candidate for preparation of new liquid crystalline materials.

\section{References}

1. Goodby JW, The nanoscale engineering of nematic liquid crystals for displays. Liquid Crystals, 2011, 38 (11-12), 1363-1387.

2. Goodby JW, Stephen J, Buchkremer A, From rods to knots using cyclohexane in smectic liquid crystals Cowling. Liquid Crystals, 2011, 38 (8), 1057-1067.

3. Barbera J, Bardajı M, Jimenez J, Laguna A, Martinez MP, Oriol L, Serrano JL, Zaragozano I, Columnar Mesomorphic Organizations in Cyclotriphosphazenes. Journal of American Chemical Society, 2005, 127, 8994-9002.

4. Jimenez J, Laguna A, Molter AM, Serrano JL, Barbera J, Oriol L, Supermolecular Liquid Crystals with a Six-Armed Cyclotriphosphazene Core: From Columnar to Cubic Phases. Chemistry Europen Journal, 2011, 17, 1029-1039.

5. Moriya K, Mizusaki H, Kato M, Yano S, Kajiwara M, Liquid crystalline phase transitions in hexakis(4-(4'-heptyloxy)biphenoxy)cyclotriphosphazene. Liquid Crystals. 1995, 18, 795800 .

6. Levelut AM, Moriya K, Structure of the liquid crystalline state in hexakis (4-(4'-alkyloxy)biphenoxy)cyclotriphosphazenes. Liquid Crystals, 1996, 20 (2), 119-124.

7. Moriya K, Suzuki T, Mizusaki H, Yano S, Kajiwara M, Liquid Crystalline Phase Transition in Organophosphazenes with 4-Octyloxybiphenyl Mesogenic Groups. Chemistry Letters, 1997, 1001-1002.

8. Moriya K, Mizusaki H, Kato M, Suzuki T, Yano S, Kajiwara M, Tashiro K, Thermal and Structural Study on Liquid-Crystalline Phase Transition in Hexakis(4-(4'-alkyloxy)biphenoxy)cyclotriphosphazene. Chemistry Materials, 1997, 9, 255-263.

9. Moriya K, Masuda T, Suzuki T, Yano S, Kajiwara M, Liquid Crystalline Phase Transition in Hexakis (4-(N-(4 ${ }^{1}$-alkoxyphenyl) iminomethyl) phenoxy) cyclotriphosphazene. Molecular Crystals and Liquid Crystals Science and Technology Section A. Molecular Crystals and Liquid Crystals, 1998, 318 (1), 267-278.

10. Moriya K, Suzuki T, Kawanishi Y, Masuda T, Mizusaki H, Nakagawa S, Ikematsu H, Mizuno K, Yano S, Kajiwara M, Liquidcrystalline phase transition in organophosphazenes. Applied Organomettalic Chemistry, 1998, 12, 771-779.

11. Moriya K, Suzuki T, Yano S, Kajiwara M, Liquid crystalline phase transitions in cyclotriphosphazenes with different mesogenic moieties in the side chains. Liquid Crystals, 1995, 19, 711713. 
12. Davarc1 D, Besli S, Demirbas E, Sythesis of a series of triplebridged cyclotriphosphazene hexa-alkoxy derivatives and investigation of their structural and mesomorphic properties, Liquid Crystals, 2013, 40(5), 624-631.

13. Jiménez J., Pintre I., Gascón E., Somolinos C. S., Alcalá R., Cavero E., Serrano J. L., Oriol L., Photoresponsive Liquid-Crystalline Dendrimers Based on Cyclotriphosphazene Core, Macromolecular Chemistry and Physics, 2014, 215, 1551-1562.

14. Bushby RJ, Kawata K, Liquid crystals that affected the world: discotic liquid crystals. Liquid Crystals, 2011; 38 (11-12): 14151426.

15. Serrano JL. Metallomesogens: Synthesis, properties and applications. New York: Wiley-VCH; 1996.

16. Moriya K, Mizusaki H, Kato M, Suzuki T, Yano S, Kajiwara M, Tashiro Y, Mesogenicity of organophosphazenes: The Effect of phosphazene rings and side Groups on the phase transition. Phosphorus, Sulfur and Silicon, 2002, 177, 1427-1432.
17. Collings PJ, Hird M. Introduction of liquid crystals chemistry and physics. Taylor and Francis, New York, 1997.

18. Goodby JW, Demus D, Gray GW, Spiess HW, Vill V. Handbook of liquid crystals. Vol. 1, Fundamentals. Wiley-VCH, Weinheim, 1998.

19. $\mathrm{Xu}$ J, Ling $\mathrm{TC}, \mathrm{He} \mathrm{C}$, Hydrogen bond-directed self-assembly of peripherally modified cyclotriphosphazenes with a homeotropic liquid crystalline phase. Journal of Polymer Science Part A: Polymer Chemistry, 2008, 46, 4691-4703.

20. Chang-Zhi Li, Matsuo Y, Nakamura E, Luminescent bow-tieshaped decaaryl[60]fullerene mesogens. Journal of American Chemical Society Committee, 2009, 131, 17058-17059. 